Pacific Journal of Mathematics

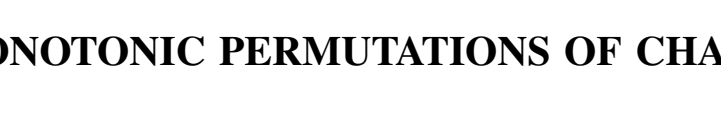




\section{MONOTONIC PERMUTATIONS OF CHAINS}

\section{Thomas J. ScotT}

An automorphism (opp) of a chain $\Omega$ is a permutation $g$ of $\Omega$ which preserves order in the sense that $\omega<\tau$ iff $\omega g<\tau g$. An anti-automorphism (orp) is a permutation $k$ of $\Omega$ which reverses order in the sense that $\omega<\tau$ iff $\omega k>\tau k$. A permutation which either preserves or reverses order is called monotonic, and the group of all monotonic permutations is denoted by $M(\Omega) . \quad M(\Omega)$ is ordered pointwise, i.e., $g \leqq h$ iff $\omega g \leqq$ $\omega$ for all $\omega \in \Omega$. This yields a $p o$-set but not a $p o$-group. However the subgroup $A(\Omega)$ of all opps of $\Omega$ forms a latticeordered group (l-group). A subgroup $K$ of $M(\Omega)$ is called $l$-monotonic if $K^{\prime}=K \cap A(\Omega)$ is nonempty, i.e., if $K$ contains an orp, and if $G(K)=K \cap A(\Omega)$ is a transitive $l$-subgroup of $A(\Omega)$. The group $M(\Omega)$ is $l$-monotonic iff $\Omega$ is homogeneous and admits an orp. The opp group $G(K)$ has index 2 in $K$ and is 0 -isomorphic to $K^{\prime}$. Thus $K^{\prime}$ is also a lattice and there exist orps $k$ in $K^{\prime}$ such that $k^{2}=1$. The stabilizer of a point $\alpha \in \Omega$ is $M_{\alpha}=\{m \in M \mid \alpha m=\alpha\}$, and the paired orbit of $\Delta$ is $\Delta^{\prime}=\{\alpha g \mid \alpha \in \Delta g$ for some $g \in G\}$. The Main Theorem 8 shows that a $K_{\alpha}$-orbit is the union of a $G_{\alpha}$-orbit and its paired $G_{\alpha}$-orbit.

An $l$-subgroup $H$ of $A(\Omega)$ is extendable if there exists an $l$-monotonic group $(K, \Omega)$ such that $G(K)=H$. Regular abelian opp groups and full periodically 0 -primitive groups are uniquely extendable. There exist both extendable and nonextendable 0 -2-transitive groups. A characterization of $o$-primitive $l$-monotonic groups is given.

The transitivity of $G(K)$ forces all $(G(K))_{\alpha}$ 's to be conjugate in $G(K)$, and also forces all $K_{\alpha}$ 's to be conjugate in $G(K)$, so that most statements about these stabilizer subgroups are independent of the choice of $\alpha$. Transitive $l$-subgroups of $A(\Omega)$ have been studied extensively by Holland [3], [4], and [5]; Lloyd [6]; and McCleary [7], [8], [9], and [10]. Standard results about po-groups and l-groups can be found in [1], while standard results about permutation groups can be found in [12]. We make minimal use of these results since the main theme of this paper is the interplay between orps and opps.

2. Basic structure theory. Let $\Omega$ be a totally ordered set (chain) containing more than one point. Points of $\Omega$ will be denoted by lower case Greek letters; subsets, by upper case Greek letters; and permutations, by lower case Roman letters. The image of $\beta \in \Omega$ under the permutation $f$ will be denoted by $\beta f$, so that if $g$ is also a permutation, $\beta(f g)=(\beta f) g$. 
Since $\Omega$ is totally ordered, a permutation $g$ is automatically an opp (orp) provided only that $\omega<\tau$ implies $\omega g<\tau g(\omega g>\tau g)$ for all $\omega, \tau \in \Omega$. If $k$ and $m$ are orps of $\Omega$ and $a$ and $b$ are opps of $\Omega$, the following facts are easily verified:

(1) $a b, \mathrm{~km}$, and $a^{-1}$ are opps;

(2) $a k, k a$, and $k^{-1}$ are orps.

It follows from these facts that $M(\Omega)$ is actually a group under composition, and that $A(\Omega)$ is a subgroup. It is well known that $A(\Omega)$ is a lattice-ordered group under the pointwise order, with $\beta(f \vee g)=\max \{\beta f, \beta g\}$ and $\beta(f \wedge g)=\min \{\beta f, \beta g\} . \quad$ A group $G$ is called a po-group iff $G$ is a po-set such that $a, b, c, d \in G$ with $b \leqq c$ implies $a b d \leqq a c d . \quad M(\Omega)$ is not a po-group, for if $b \leqq c$ are opps of $\Omega$ and $k$ is an orp of $\Omega$, then $b k \geqq c k$.

If $\Omega$ is equipped with the order topology, then it is clear that the orps and opps of $\Omega$ are homeomorphisms of $\Omega$. There exist orps of the integers without fixed points, but if $k$ is an orp of a chain $\Omega$ such that $(\alpha, \alpha k)$ is connected, since the continuous image of connected set is connected, $k$ has a fixed point in $(\alpha, \alpha k)$.

The intial number of a cardinal number is the smallest ordinal number of that cardinality. An ordinal number $\omega_{\beta}$ is regular if it is an initial number and all of its cofinal subsets have cardinality $\boldsymbol{\aleph}_{\beta}$. Following [9] we say that a point $\alpha \in \Omega$ has character $c_{\beta r}$ if $\omega_{\beta}$ is the unique regular ordinal which is $o$-isomorphic to a cofinal subset of $\{\sigma \in \Omega \mid \sigma<\alpha\}$ (or equivalently, if $\boldsymbol{\aleph}_{\beta}$ is the smallest cardinality of any cofinal subset of $\{\sigma \in \Omega \mid \sigma<\alpha\})$, and dually for $\omega_{r}$. A chain is homogeneous if $A(\Omega)$ is transitive. A point of $\Omega$ has symmetric characters if its left character equals its right character. A necessary condition for a homogeneous chain $\Omega$ to admit an orp is that points of $\Omega$ have symmetric characters. Examples of chains with nonsymmetric characters are easy to produce, e.g., the semi-long line with points of character $c_{01}$.

In the sequel all chains will be homogeneous and will admit orps. A monotonic group $(K, \Omega)$ is $t$-monotonic if $G(K)$ is transitive on $\Omega$.

THEorem 1. If $(K, \Omega)$ is monotonic, then $(K: G(K))=2$, so that $G(K)$ is normal in $K$.

Proof. It follows from facts (1) and (2) that if $k, m \in K^{\prime}, \mathrm{km}^{-1}$ is an opp. Since $G=G(K)$ is the group of all opps in $K, \mathrm{~km}^{-1}$ is in $G$, so that $G k=G m$. Hence $(K: G)=2$.

THEOREM 2. If $(K, \Omega)$ is t-monotonic, then for any $\alpha \in \Omega,\left(K_{\alpha}\right.$ : $\left.(G(K))_{\alpha}\right)=2$. 
Proof. Since $G=G(K)$ is transitive and $K$ contains at least one orp $k$, if $\alpha \in \Omega$, there exists $g \in G$ such that $\alpha k g=\alpha$. Thus $K_{\alpha}^{\prime}$ is not empty. The result now follows from a proof analogous to the proof of Theorem 1.

An opp group $(G, \Omega)$ is called regular if $G$ is transitive and $G_{\alpha}=$ $\{1\}$ for one (and hence, every) $\alpha \in \Omega$.

CoRollary 3. If $(K, \Omega)$ is monotonic, $G(K)$ is regular and $\alpha \epsilon$ $\Omega$, then $K_{\alpha}^{\prime}$ contains precisely one element.

THeOREM 4. If $(K, \Omega)$ is monotonic and $G=G(K)$, then left multiplication by a fixed orp $r \in K^{\prime}$ provides an o-isomorphism (order preserved both ways) from $G$ onto $K^{\prime}$.

Proof. If $k \leqq m \in M(\Omega)$ and $p$ is any permutation of $\Omega, \alpha p k \leqq$ $\alpha p m$ and $\alpha p^{-1} k \leqq \alpha p^{-1} m$. Thus $p k \leqq p m$ and $p^{-1} k \leqq p^{-1} m$. It follows from Theorem 1 that if $r \in K^{\prime}, r G=K^{\prime}$ and $r K^{\prime}=r^{2} G=G$. Thus if $g, h \in G, g \leqq h$ iff $r g \leqq r h$; and similarly if $k, m \in K^{\prime}, k \leqq m$ iff $r k \leqq r m$. Thus left multiplication by $r$ is an $o$-isomorphism from $G$ onto $K^{\prime}$.

CoRollary 5. If $(K, \Omega)$ is monotonic and $G(K)$ is an l-subgroup of $A(\Omega)$, then $K^{\prime}$ is a lattice with $\alpha(k \wedge m)=\min \{\alpha k, \alpha m\}$, and dually for suprema.

Proof. Since every o-isomorphism of a lattice is a lattice isomorphism, the first statement follows from Theorem 4. If $k, m \in K^{\prime}$, by Theorem $1, m=k a$ for some $a \in G(K)$. Since $1 \wedge a \in G(K)$, it follows from Theorem 4 that $k(1 \wedge a)=k \wedge m \in K^{\prime}$. If $\alpha \in \Omega, \alpha(k \wedge$ $m)=\alpha k(1 \wedge a)$, and since $\alpha k \in \Omega$ (and $1 \wedge a \in G(K)$ where infs are pointwise), $\alpha k(1 \wedge \alpha)=\alpha k \wedge \alpha k a=\min \{\alpha k, \alpha m\}$. A dual argument shows that $\alpha(k \vee m)=\max \{\alpha k, \alpha m\}$.

COROLlaRY 6. When ordered pointwise, the orps of any chain (homogeneous or not) form a lattice.

Proof. $A(\Omega)$ is an $l$-permutation group.

The following lemma uses the lattice properties of $M(\Omega)$ to establish the existence of orps which square to the identity. These orps will be very useful in $\S 3$, and in the upcoming example which shows that this nice behavior is not valid for $t$-monotonic groups.

Lemma 7. If $k$ is an orp of any chain $\Omega$, then $\left(k \wedge k^{-1}\right)^{2}=1=$ 
$\left(k \vee k^{-1}\right)^{2}$

Proof. If $k$ is an orp of $\Omega, M(\Omega)$ is monotonic so that $k \wedge k^{-1} \in$ $M^{\prime}$ by Corollary 5. If $\beta \in \Omega$ and $\beta k^{-1} \leqq \beta k$, since $k^{-1}$ is an orp, $\beta k^{-2} \geqq \beta$. Thus by Corollary $5, \beta\left(k \wedge k^{-1}\right)^{2}=\beta k^{-1}\left(k \wedge k^{-1}\right)=\min \{\beta$, $\left.\beta k^{-2}\right\}=\beta$. Similarly if $\beta k \leqq \beta k^{-1}$, we have $\beta\left(k \wedge k^{-1}\right)^{2}=\beta$ so that $\left(k \wedge k^{-1}\right)^{2}=1$. The dual argument shows that $\left(k \vee k^{-1}\right) 2=1$.

If $(G, \Omega)$ is a transitive $l$-permutation group and $\delta \in \Omega$, the $G_{\alpha^{-}}$ orbit containing $\delta$ is $\left\{\delta g \mid g \in G_{\alpha}\right\}$. It is easy to show [7, Proposition $1]$ that the orbits of $G_{\alpha}$ are convex. Thus the $G_{\alpha}$-orbits partition $\Omega$ into convex subsets, and this set inherits the natural total order, i.e., if $\Delta$ and $\Lambda$ are $G_{\alpha}$-orbits, then $\Delta \leqq \Lambda$ iff $\delta \leqq \gamma$ for all $\delta \in \Delta, \gamma \in \Lambda$. Furthermore, this natural total order is independent of $\alpha$ [7, Theorem 9]. We define for each $G_{\alpha}$-orbit $\Delta$, a paired orbit $\Delta^{\prime}=\{\alpha g \mid \alpha \in \Delta g\}$, and always use the notation $\Delta^{\prime}$ to refer to pairings with respect to some distinguished point $\alpha$. It is shown in [12, §16] and [7, Theorem 9] that $\Delta^{\prime}$ is indeed a $G_{\alpha}$-orbit, and in [7, Proposition 4] that the map $\Delta \rightarrow \Delta^{\prime}$ is an 0 -anti-isomorphism of the set of $G_{\alpha}$-orbits with the property that $\Delta^{\prime \prime}=\Delta$ for any $G_{\alpha}$-orbit $\Delta$.

If $\beta \in \Omega$ and $\beta G_{\alpha}=\{\beta\}$, then $\beta$ is called a fixed point of $G_{\alpha}$. If $\beta G_{\alpha} \neq\{\beta\},\left\{\beta G_{\alpha}\right\}$ is a long $G_{\alpha}$-orbit which must necessarily be infinite. A $G_{\alpha}$-orbit $\Delta$ is called positive (negative) iff $\delta>\alpha(\delta<\alpha)$ for each $\delta \in \Delta$, and $\{\alpha\}$ is called the zero $G_{\alpha}$-orbit.

The following theorem describes the relationship between the $G(K)_{\alpha}$-orbits and the $K_{\alpha}$-orbits of an $l$-monotonic group $(K, \Omega)$.

Theorem 8 (Main Theorem). If $(K, \Omega)$ is l-monotonic, $G=G(K)$, $\Delta$ is a $G_{\alpha}$-orbit, and $k \in K_{\alpha}^{\prime}$, then $\Delta k=\Delta^{\prime}$. Thus a $K_{\alpha}$-orbit is the union of a $G_{\alpha}$-orbit and its paired $G_{\alpha}$-orbit.

Proof. If $k, m \in K_{\alpha}^{\prime}$, since $k m^{-1} \in G_{\alpha}, \Delta k m^{-1}=\Delta$ and $\Delta k=\Delta m$. It follows that $\Delta K_{\alpha}^{\prime}$ is the union of $G_{\alpha}$-orbits, for suppose that $\Gamma$ is a $G_{\alpha}$-orbit not contained in $\Delta k$ which meets $\Delta k$. Then if $\gamma \in \Gamma \backslash \Delta k$ and $\beta \in \Gamma \cap \Delta k$, there exists $g \in G_{\alpha}$ such that $\beta g=\gamma$. But then $\Delta k g \neq \Delta k$, which is a contradiction since $k g \in K_{\alpha}^{\prime}$. Thus $\Delta k$ is the union of $G_{\alpha^{-}}$. orbits.

Suppose $\delta k, \tau k \in \Delta k$. Then since $\Delta$ is a $G_{\alpha}$-orbit, there exists $g \in$ $G_{\alpha}$ such that $\delta g=\tau$. Thus $\delta k\left(k^{-1} g k\right)=\delta g k=\tau k$, and since $k^{-1} g k \in G_{\alpha}$, $\delta k$ and $\tau k$ are in the same $G_{\alpha}$-orbit. This shows that $\Delta k$ is a $G_{\alpha}$-orbit.

We note that both pairing and $k \in K_{\alpha}^{\prime}$ provide an involution of the set of $G_{\alpha}$-orbits, so that we may assume that $\Delta$ is a positive $G_{\alpha^{-}}$ orbit. To show $\Delta k=\Delta^{\prime}$, first suppose $\Delta^{\prime}<\Delta k$. 


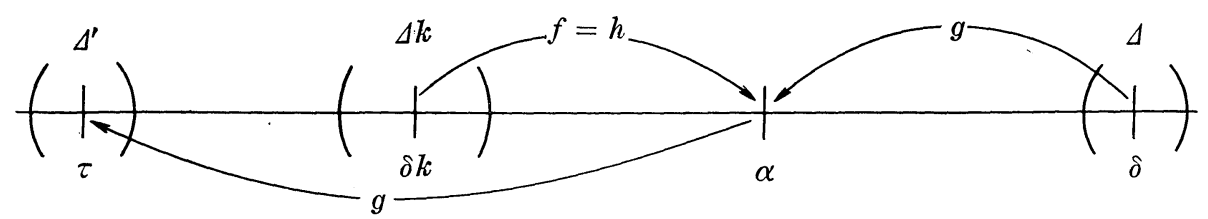

If $\delta \in \Delta$, there exists $g \in G$ such that $\delta g=\alpha$, and from the definition of pairing we have $\alpha g=\tau \in \Delta^{\prime}$ so that $\alpha g<\delta k$. But $G$ is a transitive $l$-group, so there exists $h \in G$ such that $\delta k h=\alpha$. Now $g^{-1} \geqq g^{-1} \wedge$ $h=f$ and $\alpha=\delta k h=\delta k f$; also $\alpha k g^{-1} k f=\alpha g^{-1} k f=\delta k f=\alpha$. But since $g^{-1} \geqq f, \delta k g^{-1}>\delta k f=\alpha$, so that $\delta k g^{-1} k f<\alpha f$. Since pairing is an orp of the $G_{\alpha}$-orbits and $\Delta k>\Delta^{\prime},(\Delta k)^{\prime}<\Delta^{\prime \prime}=\Delta$. Since $(\delta k) f=$ $\alpha$, from the definition of pairing we have $\alpha f \in(\Delta k)^{\prime}<\Delta$. Thus $\delta k g^{-1} k f<\alpha f \in(\Delta k)^{\prime}<\Delta$, and this is a contradiction since $k g^{-1} k f \in G_{\alpha}$ and $\Delta$ is a $G_{\alpha}$-orbit.

If $\Delta k<\Delta^{\prime}$ a dual argument leads to a contradiction. This completes the proof of Theorem 8 .

Corollary 9. If $(K, \Omega)$ is l-monotonic and $G=G(K)$, the paired $G_{\alpha}$-orbits are o-anti-isomorphic.

Proof. The $o$-anti-isomorphism is achieved by means of any $k \in K_{\alpha}^{\prime}$.

Transitive $l$-subgroups $G$ of $A(\Omega)$ such that fixed points of $G_{\alpha}$ are never paired with long $G_{\alpha}$-orbits were called balanced in [7]. Examples of unbalanced $l$-permutation groups can be constructed, but it follows from Corollary 9 that if $(K, \Omega)$ is $l$-monotonic, then $G(K)$ is balanced.

A monotonic group $(K, \Omega)$ is called $c$-monotonic if $G(K)$ is coherent, i.e., $\alpha<\beta \in \Omega$ implies that there exists $1 \leqq g \in G(K)$ such that $\alpha g=$ $\beta$. The chain of implications $l$-monotonic $\Rightarrow c$-monotonic $\Rightarrow t$-monotonic $\Rightarrow$ monotonic is easy to verify. The following is an example of a $c$-monotonic group $(K, \Omega)$ which not only has no orp $k$ such that $k^{2}=1$ but also does not have the orbit pairing property of Theorem 8 .

Suppose $H$ is the subgroup of the linear group of the reals which consists of the elements $\{\alpha x+\beta \mid \alpha$ is a positive rational and $\beta$ is any real number\}. An element $g$ of $H$ is positive iff $\alpha=1$ and $\beta>0$. Then $H$ is a coherent opp group, but not an $l$-permutation group. Let $k$ be the orp of the reals which sends each real number $\alpha$ to $-\sqrt{2} \alpha$. Since $\alpha k^{2}=2 \alpha, k^{2} \in H$. If $h \in H$ there is a positive rational number $\tau$ and a real number $\beta$ such that $\alpha h=\tau \alpha+\beta$ for each real number $\alpha$. Hence $\alpha k h k=2 \tau \alpha-\sqrt{2} \beta$, so that $k h k \in H$ for each $h \epsilon$ $H$. The element $g$ of $H$ defined by $\tau g=\tau / 2$ has the property that $k^{-1}=k g$, so that $k h k^{-1} \in H$ for each $h \in H$. Since $k^{2} \in H$ if $K=\langle k, H\rangle$, it is easy to show that $G(K)=H$. (In fact this result follows from Theorem 10.) Thus $K$ is $c$-monotonic, and if $m \in K_{0}^{\prime}$, there exists a 
positive rational number $\gamma$ such that $\alpha m=-\sqrt{2} \gamma \alpha$ for each $\alpha$. Since $\alpha m^{2}=2 \gamma^{2} \alpha$ and $\sqrt{2}$ is irrational, $m^{2} \neq 1$.

If $r \in K^{\prime}$ there is a positive rational number $\eta$ and a real number $\lambda$ such that $\alpha r=-\sqrt{2} \eta \alpha+\lambda$ for each $\alpha$. But then if $r^{2}=1$, since $0=0 r^{2}=\lambda(1-\sqrt{2} \eta)$, either $\lambda=0$ or $\sqrt{2}$ is rational, and because of the above, both of these statements lead to contradictions. Thus no orp in $K$ squares to the identity.

Since the positive rationals are an orbit of $H_{0}$, the orbits of $H_{0}$ are not convex; and furthermore, since the positive rationals are paired (in $H_{0}$ ) with the negative rationals, $K$ clearly does not have the orbit pairing property of Main Theorem 8.

3. Extendable $l$-permutation groups. If $H$ is an $l$-subgroup of $A(\Omega)$, an orp of $k$ of $\Omega$ will be said to extend $H$ iff $G(\langle k, H\rangle)=H$. If $k$ extends $H$, we note that for any $a \in H, k a$ also extends $H$. If $(K, \Omega)$ is $l$-monotonic and $G(K)=H, K$ will be called an extension of $H$, and $H$ will be called extendable. The following theorem provides a computational necessary and sufficient condition for extendability.

THEOREM 10. An orp $m$ extends an l-subgroup $H$ of $A(\Omega)$ iff $m$ normalizes $H$ and $m^{2} \in H$.

Proof. If $H$ is extendable, any $m \in K \backslash H$ normalizes $H$, and clearly, $m^{2} \in H$.

Conversely, if such an orp $m$ exists, $m g m^{-1} \in H$ for each $g \in H$, so that since $m^{2} \in H, m g m=m g m^{-1} m^{2} \in H$. Similarly $m^{-1} g m^{-1} \in H$. Since only words which contain an even number of $m^{\text {'s }}$ or $m^{-1}$ 's are opps, it follows that $m$ extends $H$.

Theorem 11. Suppose that $(H, \Omega)$ is regular. Then $H$ is extendable iff $H$ is abelian; and then $H$ uniquely determines its extension.

Proof. If $K$ is an extension of $H$ and $H=G(K)$ is regular, we know by Corollary 3 that there is precisely one $k \in K_{\alpha}^{\prime}$. Since each $\beta \in \Omega$ is an $H_{\alpha}$-orbit, we know by Main Theorem 8 and the definition of pairing that $\beta k=\beta^{\prime}=\alpha g$, where $g$ is the unique element of $H$ such that $\beta g=\alpha$. We next show that this orp $k$ extends $H$ iff $H$ is abelian.

Since $k$ fixes $\alpha, k^{2}$ fixes $\alpha$ so that by regularity, $k^{2}=1$ and $k=$ $k^{-1}$. It suffices (by Theorem 10) to show that $k g k \in H$ for each $g \in H$.

From the definition of $k$ we have $\alpha k g k=\alpha g k=(\alpha g)^{\prime}=\alpha g^{-1}$ for any $g$ in $H$. If $\beta \in \Omega$, there is a unique $h$ in $H$ such that $\beta h=\alpha$. 
Thus $\beta k g k=\alpha h g k=(\alpha h g)^{\prime}=\alpha g^{-1} h^{-1}$. But since $H$ is abelian $\alpha g^{-1} h^{-1}=$ $\alpha h^{-1} g^{-1}=\beta g^{-1}$. Thus for each $g \in H$, we have $k g k=g^{-1}$ so that $k$ extends $H$. Also $H$ uniquely determines its extension, since $k$ must belong to any extension of $H$, and thus, all extensions are simply extensions by $k$.

If $H$ is not abelian, by regularity there exist $c, d \in H$ such that $\gamma c^{-1} d^{-1} \neq \gamma d^{-1} c^{-1}$ for any $\gamma \in \Omega$. Then picking $\gamma$ such that $\gamma c=\alpha$, we have $\alpha k d k=\alpha d k=(\alpha d)^{\prime}=\alpha d^{-1}$; but since $\gamma c=\alpha, \gamma k d k=\alpha c d k=$ $(\alpha c d)^{\prime}=\alpha d^{-1} c^{-1} \neq \alpha c^{-1} d^{-1}=\gamma d^{-1}$. Thus $k d k$ agrees with $d^{-1}$ at $\alpha$ but not at $\gamma$; so by regularity, $k d k \notin H$. Thus since $k$ must belong to any extension of $H, H$ is not extendable.

Corollary 12. Suppose that $(K, \Omega)$ is montonic and $G=G(K)$ is regular. Then for any $m \in K^{\prime}$ and $g \in G, m^{2}=1$ and $m g=g^{-1} m$.

Proof. In the proof of Theorem 11 we actually showed that for any $h \in G, k h k=h^{-1}$ where $k$ is the only orp in $K_{\alpha}^{\prime}$. Thus $(k h)^{2}=1$ and since $k=k^{-1}, k h=h^{-1} k$. If $m \in K^{\prime}$, by Theorem $1 m=k f$ for some $f \in G$, so that $m^{2}=(k f)^{2}=1$. Now if $g \in G, m g=(k f) g$ so that since $f g \in G,(m g)^{2}=(k(f g))^{2}=1$, and since $m^{2}=1$ we have $m g=g^{-1} m$.

If $F$ is any group of permutations on $\Omega$, then a convex $F$-congruence on $Q$ is an equivalence relation $Q$ on $\Omega$ such that each $Q$ class is convex, and such that if $\alpha Q \beta$ then $\alpha f Q \beta f$ for each $f \in F$. If $L$ is any $t$-monotonic subgroup of $M(\Omega)$, it follows from the transitivity of $G(L)$ that all convex $L$-congruence classes for any one convex $L$-congruence are o-isomorphic. If $Q$ is a convex $L$-congruence, we call each $Q$-class an o-block of $L$; thus an o-block of $L$ is a nonempty convex subset $\Delta$ of $\Omega$ such that $\Delta m=\Delta$ or $\Delta m \cap \Delta=\{\}$ for each $m \in L . \quad L$ is called o-primitive iff the only convex $L$-congruences are trivial ones.

A subgroup $G$ of $A(\Omega)$ is o-2-transitive iff whenever $\alpha<\beta$ and $\gamma<\delta$, there exists $g \in G$ such that $\alpha g=\gamma$ and $\beta g=\delta$. It is clear that if $(H, \Omega)$ is an $o-2$-transitive opp group, then $H$ is o-primitive. Proposition 24 [7] states that a regular opp group $(H, \Omega)$ is o-primitive iff $H$ is isomorphic as an ordered group to a subgroup of the additive reals.

The easiest example of a transitive $l$-permutation group which is neither 0 -2-transitive nor regular is the group $(G, \Omega)$, where $\Omega$ is the reals and $G=\{g \in A(\Omega) \mid \alpha g+1=(\alpha+1) g$ for all $\alpha \in \Omega\}$. Some comments on this group will facilitate the understanding of the next few theorems. The long orbits of any $G_{\alpha}$ form a chain o-isomorphic to the integers (in fact, the long $G_{0}$-orbits are the intervals $(n, n+1)$ where $n$ is an integer). The $o$-permutation $z$ defined by $\alpha z=\alpha+1$ generates (as a group) the centralizer $Z_{A(2)} G$, and $z$ is called the $\Omega$-period of $G$. 
Because of this periodicity, the action of $g \in G$ on any long $G_{\alpha}$-orbit determines its action on all of $\Omega$. The long $G_{\alpha}$-orbit $\Delta_{j+1}$ is "one period up" from $\Delta_{j}$ in the sense that $\Delta_{j} z=\Delta_{j+1}$.

McCleary's Theorem 40 [7] states that any transitive $o$-primitive $l$-permutation group which is neither o-2-transitive nor regular looks strikingly like $(G, \Omega)$. These groups were called periodically o-primitive in [7]. Here, more precisely, is what Theorem 40 says.

Let $(G, \Omega)$ be an $o$-primitive transitive $l$-permutation group which is neither 0 -2-transitive nor regular, and let $\alpha \in \Omega$. Then the long orbits of $G_{\alpha}$ form a chain 0 -isomorphic to the integers. Suppose $\Delta_{1}=$ $\left(\Delta_{1}\right)_{\alpha}$ is the first positive long orbit of $G_{\alpha}, \Delta_{j+1}$ is the first long orbit greater than $\Delta_{j}$, and $\bar{\omega}_{j}$ is the sup of $\bar{\Delta}_{j}$. Either there is a positive integer $n$ such that $\sup \Delta_{j}=\bar{\omega} \in \Omega$ iff $j \equiv 0(\bmod n)$, and we say that $G$ has $\operatorname{Config}(n)$; or sup $\Delta_{j}=\omega_{j} \in \Omega$ only when $j=0$, and we say that $G$ has Config $(\infty)$. The o-permutation $\bar{z}$ of $\bar{\Omega}, \bar{\Omega}$ the Dedekind completion (without end points) of $\Omega$, such that $\alpha \bar{z}=\sup \left(\Delta_{1}\right)_{\alpha}=\bar{\omega}_{1}$ for each $\alpha \in \Omega$ is called the $\bar{\Omega}$-period of $G$ in the sense that it generates (as a group) the centralizer $Z_{A(\bar{\Omega})} G$; so that $(\bar{\beta} \bar{z}) g=(\bar{\beta} g) \bar{z}$ for all $\bar{\beta} \in \bar{\Omega}, g \in G$. If $G$ has $\operatorname{Config}(n), z=\bar{z}^{n}$ is called the $\Omega$-period of $G$. $G$ is called full if $G$ is the entire centralizer $Z_{A(\bar{\Omega})} \bar{z}$.

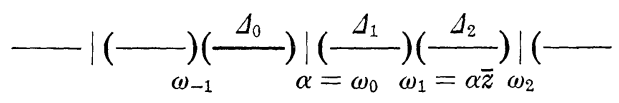

CONFIG (2)

Lemma 13. Suppose that $(F, \Omega)$ is a periodically o-primitive l-permutation group and $t$ is either the $\Omega$-period or the $\bar{\Omega}$-period of $F$. Then if an orp $k$ extends $F, t k=k t^{-1}$, i.e., if $\beta$ is one period up from $\gamma, \beta k$ is one period down from $\gamma k$. Conversely if $(H, \Omega)$ is full periodically o-primitive with $t$ either period of $H$, and $k$ is an orp of $\Omega$ such that $t k=k t^{-1}$, then $k$ extends $H$.

Proof. Suppose $t$ is the $\bar{\Omega}$-period of $F$. If $k$ extends $F$, then for some $a \in F, m=k \alpha$ fixes $\alpha$. Since $\bar{\omega}_{n}$ is fixed by $F_{\alpha}$ for each integer $n$, it follows from Theorem 8 that $\bar{\omega}_{n} m=\bar{\omega}_{-n}$. Thus $\alpha t m=$ $\bar{\omega}_{1} m=\bar{\omega}_{-1}=\alpha t^{-1}=\alpha m t^{-1}$. If $\beta \in \Omega$, since $F$ is transitive, $\beta f=\alpha$ for some $f \in F$. Then $\beta f m t^{-1}=\alpha m t^{-1}=\alpha t m=\beta f t m=\beta t f m$ since $t$ centralizes $F$. Since $k$ (and hence $m$ ) extends $F$, there exists $c \in F$ such that $f m=m c$. But then $\beta f m t^{-1}=\beta m c t^{-1}=\beta m t^{-1} c$, and since also $\beta f m t^{-1}=\beta t f m=\beta t m c$, we have $\beta t m=\beta m t^{-1}$. Thus $t m=m t^{-1}$ and since $m=k a$, we have $t k a=k a t^{-1}=k t^{-1} a$ so that $t k=k t^{-1}$ as desired.

Conversely suppose $(H, \Omega)$ is full and $t$ is the $\bar{\Omega}$-period of $H$. If $k$ is an orp such that $t k=k t^{-1}$, it follows that $t^{-1} k=k t$ and $t^{-1} k^{-1}=k^{-1} t$. 
Since $H$ is full, using Theorem $10, k$ extends $H$ iff for each $g$ in $H$, $k g k^{-1}, k^{-1} g k$, and $k^{2}$ all commute with $t$. If $g \in H, k g k^{-1} t=k g t^{-1} k^{-1}=$ $t k g k^{-1}$. Thus $k g k^{-1}$ (and similarly $k^{-1} g k$ and $k^{2}$ ) commutes with $t$. Thus $k$ extends $H$. The proof for the corresponding $\Omega$-period is similar.

Lemma 14. Suppose $(H, \Omega)$ is periodically o-primitive with finite Config $(n), \Delta_{i}$ is the $i$ th positive $H_{\alpha}$-orbit, and $\Psi=\Delta_{1} \cup \cdots \cup \Delta_{n}$. Then $\Omega$ has an orp iff $\Psi$ has an orp.

Proof. Since $H$ has $\operatorname{Config}(n)$ if $z$ is the $\Omega$-period of $H, \alpha z \in \Omega$ so that if $m$ is an orp of $\Omega, \alpha<\alpha z$ and $\alpha z m<\alpha m$. Since $H$ is periodically 0 -primitive, $A(\Omega)$ is 0 -primitive. Since $A(\Omega)$ is not periodic [6], it must be 0 -2-transitive. Thus there is a $g$ in $A(\Omega)$ such that $\alpha z m g=\alpha$ and $\alpha m g=\alpha z$. Thus $m g$ induces an orp on $\Psi$.

Since $H$ has Config $(n), z$ is actually in $A(\Omega)$. Thus if $m$ is an orp of $\Psi$, we define a function $k$ by

$$
\beta k=\left\{\begin{array}{l}
\left(\left(\beta z^{-t}\right) m\right) z^{-t-1} \text { if } \beta \in \Psi_{t}=\Psi z^{t} \\
\bar{\omega}_{-t}=\bar{\omega}_{t^{\prime}} \text { if } \beta=\bar{\omega}_{t} \in \bar{\Omega}
\end{array}\right\} .
$$

Since the long orbits and fixed points of $H_{\alpha}$ partition $\Omega$, and $m$ is an orp of $\Psi, k$ is an orp of $\Omega$ which is essentially the "period extension" of $m$ to $\Omega$.

THEOREM 15. If $(H, \Omega)$ is full periodically o-primitive with finite Config $(n)$, and $\Omega$ has an orp, then $H$ is uniquely extendable.

Proof. If $\alpha \in \Omega$ and $\Psi$ is as in Lemma 14, then by Lemma 14 $\Psi$ has an orp $m$ which we periodically extend to the orp $k$ of $\Omega$ as in Lemma 14. To show that $H$ is extendable it suffices by Lemma 13 to show that if $z$ is the $\Omega$-period of $H$, then $z k=k z^{-1}$. If $\beta \in \Delta_{t}$, $t=a n+b, 0 \leqq b<n$, then $\beta z \in \Psi_{a+1}=\Psi z^{a+1}$ so that by the definition of $k, \beta z k=(\beta z) z^{-a-1} m z^{-a-2}=\left(\beta\left(z^{-a} m z^{-a-1}\right)\right) z^{-1}=\beta k z^{-1}$. Similarly if $\beta=\omega_{a n} \in \Omega, \beta z k=\beta k z^{-1}$ so that $z k=k z^{-1}$, and thus $k$ extends $H$ by Lemma 13.

If $k$ and $r$ both extend $H$, it follows from Lemma 13 that $z k=$ $k z^{-1}$ and $z r=r z^{-1}$. By Theorem $1, r=a k$ for some $a \in A(\Omega)$, so that $z a k=z r=r z^{-1}=a k z^{-1}=a z k$, i.e., $z a=a z$. Thus $a \in H$ since $H$ is full, and it follows that $H$ is uniquely extendable.

If $\Delta$ and $\Gamma$ are subsets of a chain $\Omega$, we write $\Delta<\Gamma$ iff $\delta<\gamma$ for all $\delta \in \Delta, \gamma \in \Gamma$. Let $\alpha$ be an ordinal number. An $\alpha$-set is a chain 
$\Omega$ of cardinality $\boldsymbol{\aleph}_{\alpha}$ in which for any two (possibly empty) subsets $\Delta<\Gamma$ of cardinality less that $\boldsymbol{\aleph}_{\alpha}$, there exists $w \in \Omega$ such that $\Delta<$ $\omega<\Gamma$. If $\omega_{\alpha}$ is a regular ordinal, then (assuming the generalized continuum hypothesis) there exists an $\alpha$-set, and it is unique up to $o$ isomorphism [2, pp. 179-181]. Reversing the ordering on an $\alpha$-set yields an $\alpha$-set, so by the uniqueness of $\alpha$-sets, every $\alpha$-set possesses an orp.

It is shown in [8, Lemma 22] that if $H$ is a periodically $o$-primitive $l$-subgroup of $A(\Omega)$ and $\Delta_{1}=\left(\Delta_{1}\right)_{\beta}$ is an $\alpha$-set, then all long $H_{\beta}$-orbits $\Delta_{i}$ are $\alpha$-sets. Theorem 24 [8] states that if $n=1,2, \cdots$, or $\infty$, and $\Delta$ is an $\alpha$-set (where $\omega_{\alpha}$ is a regular ordinal number) then there exists a unique (up to o-permutation group isomorphism) full periodically $o$ primitive group $(H, \Omega)$ having $\Delta$ as the first positive orbit of a stabilizer subgroup $G_{\beta}$ and having Config $(n)$. We have

THEOREM 16. Let $n=1,2, \cdots$, or $\infty$, let $\omega_{\alpha}$ be a regular ordinal number, and let $\Delta_{1}$ be an $\alpha$-set. Then the unique full periodically o-primitive l-permutation group $(H, \Omega)$ having $\Delta_{1}$ as the first positive orbit of a stabilizer subgroup $H_{\beta}$ and having Config $(n)$ is uniquely extendable.

Proof. Suppose that $n$ is finite, $\Delta_{i}$ be the $i$ th positive orbit of $H_{\beta}$ and $\Psi=\Delta_{1} \cup \cdots \cup \Delta_{n}$. Since each $\Delta_{i}$ is an $\alpha$-set, it has an orp and furthermore, $\Delta_{i}$ is also $o$-isomorphic to $\Delta_{j}$ for any integer $j$; therefore $\Psi$ has an orp. Thus $\Omega$ has an orp by Lemma 14 and $H$ is uniquely extendable by Theorem 15 .

If $n=\infty$, one can use the $\bar{\Omega}$-period $\bar{z}$ of $H$ and a special property of $\alpha$-sets (namely Lemma 23 [8]) to show that $H$ is uniquely extendable by a proof similar to the proof of Theorem 15 .

A chain $\Omega$ is 0 -2-homogeneous iff $A(\Omega)$ is 0 -2-transitive. The support of $m \in M(\Omega)$ is $\{\beta \in \Omega \mid \beta m \neq \beta\}$. An $l$-ideal of an $l$-group $G$ is a convex normal $l$-subgroup of $G$. We make the following definitions:

$$
\begin{aligned}
B(\Omega) & =\{g \in A(\Omega) \mid g \text { has bounded support }\} \\
B A(\Omega) & =\{g \in A(\Omega) \mid g \text { has support bounded above }\} \\
B B(\Omega) & =\{g \in A(\Omega) \mid g \text { has support bounded below }\} .
\end{aligned}
$$

It is shown in [3, Theorem 6] that when $\Omega$ is 0 -2-homogeneous, $B$, $B A$, and $B B$ are all $o$-2-transitive $l$-ideals of $A(\Omega)$. We have the following theorem.

Theorem 17. Suppose that $\Omega$ is o-2-homogeneous and has an orp. Then $B(\Omega)$ is extendable, but in general, not uniquely extendable. Furthermore $B A(\Omega)$ and $B B(\Omega)$ are not extendable. 
Proof. If $m$ is any orp of $\Omega$, it is straightforward to show that $m$ fixes $\gamma$ iff $m^{-1} h m$ fixes $\gamma m$. Thus conjugation by any orp $m$ fixes $B(\Omega)$ and interchanges $B A(\Omega)$ and $B B(\Omega)$, so that $B A(\Omega)$ and $B B(\Omega)$ are never extendable. If $m$ is an orp of $\Omega$ which squares to 1 (such an orp exists by Lemma 7 since $M(\Omega)$ is $l$-monotonic), since $m^{-1} B(\Omega) m=$ $B(\Omega)$ and $m^{2}=1 \in B(\Omega)$, $m$ extends $B(\Omega)$ by Theorem 10 .

If $\Omega$ is the reals, and the orps $k, n$ of the reals are defined by: $\alpha k=-\alpha$ for each $\alpha$; and $\alpha n=-2 \alpha$ if $\alpha \geqq 0, \alpha n=-\alpha / 2$ otherwise, then both $k$ and $n$ extend $B(\Omega)$. The extensions $K=\langle k, B\rangle$ and $N=$ $\langle n, B\rangle$ are definitely not identical however, for $n$ is clearly not in $k B$.

It follows from Corollary 9 that a necessary condition for $(H, \Omega)$ to be extendable is that the paired $H_{\alpha}$-orbits be $o$-anti-isomorphic which implies that $H$ must be balanced. Balanced is not sufficient for extendability since $B A$ and $B B$ are both balanced whenever $\Omega$ is $0-2$ homogeneous.

In [11] the generalized monotonic wreath product is constructed (along the same lines as the generalized ordered wreath product constructed in [5] but different in one crucial way), and it is shown that an $l$-monotonic group can be "nicely" embedded in the generalized monotonic wreath product of its "o-primitive components". Thus a study of $o$-primitive $l$-monotonic groups is called for.

If $(K, \Omega)$ is an 0 -primitive $l$-monotonic group, $G(K)$ is either 0 -2transitive, the regular representation of a subgroup of the reals, or periodically $o$-primitive. If $G(K)$ is $o$-2-transitive, then $K$ is actually 2-transitive, i.e., if $\alpha, \beta, \gamma, \delta \in \Omega$, there exists $k \in K$ such that $\alpha k=$ $\gamma$ and $\beta k=\delta$. If $\alpha<\beta$ and $\gamma>\delta$ and $k$ is an orp, then $\alpha k>\beta k$ so there exists $g \in G(K)$ such that $\alpha k g=\gamma$ and $\beta k g=\delta$. The other cases are similar, and it follows that $K$ is 2-transitive. It is shown in [8] that when $A(\Omega)$ is 0 -2-transitive, it is actually $o$ - $n$-transitive for $n \geqq 3$. Since an orp can have at most one fixed point, $M(\Omega)$ is not 3 -transitive.

If $G=G(K)$ is the regular representation of a subgroup of the reals, Corollary 12 shows that if $k \in K^{\prime}, k^{2}=1$ and $k g=g^{-1} k$ for any $g \in G$. If $G=G(K)$ is periodically $o$-primitive with $\bar{\Omega}$-period $\bar{z}$, and $k \in K^{\prime}$, since $k$ extends $G, \bar{z} k=k \bar{z}^{-1}$ by Lemma 13 . We summarize these results in

THEOREM 18. If $(K, \Omega)$ is an o-primitive l-monotonic group and $G=G(K)$, then either:

(1) $G$ is the regular representation of a subgroup of the reals, and if $k \in K^{\prime}, g \in G, k^{2}=1$ and $k g=g^{-1} k$; or

(2) $G$ is o-2-transitive, and $K$ is 2-transitive; or

(3) $G$ is periodically o-primitive with $\bar{\Omega}$-period $\bar{z}$, and $\bar{z} k=k \bar{z}^{-1}$ for any $k \in K^{\prime}$. 


\section{REFERENCES}

1. L. Fuchs, Partially Ordered Algebraic Systems, Addison-Wesley, Reading, Mass., 1963.

2. F. Hausdorff, Grundzuge der Mengenlehre, Veit and Co., Leipzig, Germany, 1914.

3. C. Holland, The lattice-ordered group of automorphisms of an ordered set, Michigan Math. J., 10 (1963), 399-408.

4. - Transitive lattice-ordered permutation groups, Math. Zeit., 87 (1965), 420-433.

5. C. Holland and S. H. McCleary, Wreath products of ordered permutation groups, Pacific J. Math., 31 (1969), 703-716.

6. J. T. Lloyd, Lattice-ordered Groups and o-permutation Groups, Dissertation, Tulane University, 1964.

7. S. H. McCleary, O-primitive ordered permutation groups, Pacific J. Math., 40 (1972).

8. - O-primitive ordered permutation groups $I I$, (to appear).

9. - The lattice-ordered group of automorphisms of an $\alpha$-set, (to appear).

10. - Generalized wreath products viewed as sets with valuation, J. Algebra, 16 (1970), 163-182.

11. T. J. Scott, Monotonic Permutations of Chains, Dissertation, The University of Georgia, 1972.

12. H. Wielandt, Finite Permutation Groups, Academic Press, New York, N. Y., 1964.

Received March 12, 1974. This paper grew out of the author's doctoral dissertation written under Steve McCleary. It was supported in part by the Georgia College Faculty Research Fund.

Georgia College 


\section{PACIFIC JOURNAL OF MATHEMATICS}

\section{EDITORS}

RICHARD ARENS (Managing Editor)

University of California

Los Angeles, California 90024

\section{R. A. Beaumont \\ University of Washington \\ Seattle, Washington 98105}

\section{J. DugundJI}

Department of Mathematics

University of Southern California

Los Angeles, California 90007

D. Gilbarg and J. Milgram

Stanford University

Stanford, California 94305

\section{ASSOCIATE EDITORS}
E. F. BECKENBACH
B. H. NeumanN
F. WOLF
K. YOSHIDA

\section{SUPPORTING INSTITUTIONS}

UNIVERSITY OF BRITISH COLUMBIA
CALIFORNIA INSTITUTE OF TECHNOLOGY
UNIVERSITY OF CALIFORNIA
MONTANA STATE UNIVERSITY
UNIVERSITY OF NEVADA
NEW MEXICO STATE UNIVERSITY
OREGON STATE UNIVERSITY
UNIVERSITY OF OREGON
OSAKA UNIVERSITY

UNIVERSITY OF BRITISH COLUMBIA

UNIVERSITY OF CALIFORNIA

MONTANA STATE UNIVERSITY

NEW MEXICO STATE UNIVERSITY

OREGON STATE UNIVERSITY

OSAKA UNIVERSITY

\author{
UNIVERSITY OF SOUTHERN CALIFORNIA \\ STANFORD UNIVERSITY \\ UNIVERSITY OF TOKYO \\ UNIVERSITY OF UTAH \\ WASHINGTON STATE UNIVERSITY \\ UNIVERSITY OF WASHINGTON \\ AMERICAN MATHEMATICAL SOCIETY \\ NAVAL WEAPONS CENTER
}

The Supporting Institutions listed above contribute to the cost of publication of this Journal, but they are not owners or publishers and have no responsibility for its content or policies.

Mathematical papers intended for publication in the Pacific Journal of Mathematics should be in typed form or offset-reproduced, (not dittoed), double spaced with large margins. Underline Greek letters in red, German in green, and script in blue. The first paragraph or two must be capable of being used separately as a synopsis of the entire paper. Items of the bibliography should not be cited there unless absolutely necessary, in which case they must be identified by author and Journal, rather than by item number. Manuscripts, in triplicate, may be sent to any one of the editors. Please classify according to the scheme of Math. Reviews, Index to Vol. 39. All other communications should be addressed to the managing editor, or Elaine Barth, University of California, Los Angeles, California, 90024.

The Pacific Journal of Mathematics expects the author's institution to pay page charges, and reserves the right to delay publication for nonpayment of charges in case of financial emergency.

100 reprints are provided free for each article, only if page charges have been substantially paid. Additional copies may be obtained at cost in multiples of 50 .

The Pacific Journal of Mathematics is issued monthly as of January 1966. Regular subscription rate: $\$ 72.00$ a year (6 Vols., 12 issues). Special rate: $\$ 36.00$ a year to individual members of supporting institutions.

Subscriptions, orders for back numbers, and changes of address should be sent to Pacific Journal of Mathematics, 103 Highland Boulevard, Berkeley, California, 94708.

PUBLISHED BY PACIFIC JOURNAL OF MATHEMATICS, A NON-PROFIT CORPORATION

Printed at Kokusai Bunken Insatsusha (International Academic Printing Co., Ltd.), 270, 3-chome Totsuka-cho, Shinjuku-ku, Tokyo 160, Japan.

Copyright (C) 1973 by Pacific Journal of Mathematics Manufactured and first issued in Japan 


\section{Pacific Journal of Mathematics \\ Vol. 55, No. $2 \quad$ October, 1974}

Walter Allegretto, On the equivalence of two types of oscillation for elliptic

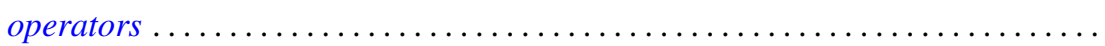

Edward Arthur Bertram, A density theorem on the number of conjugacy classes in

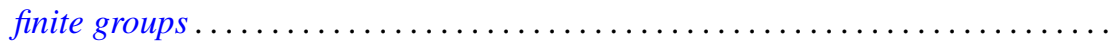

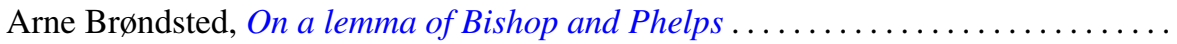

Jacob Burbea, Total positivity and reproducing kernels ..................

Ed Dubinsky, Linear Pincherle sequences . . . . . . . . . . . . . . . . . .

Benny Dan Evans, Cyclic amalgamations of residually finite groups .............

361

Barry J. Gardner and Patrick Noble Stewart, A "going down" theorem for certain

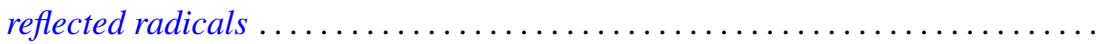

381

Jonathan Light Gross and Thomas William Tucker, Quotients of complete graphs:

revisiting the Heawood map-coloring problem ....................

Sav Roman Harasymiv, Groups of matrices acting on distribution spaces .........

Robert Winship Heath and David John Lutzer, Dugundji extension theorems for

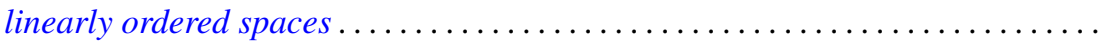

Chung-Wu Ho, Deforming p. l. homeomorphisms on a convex polygonal

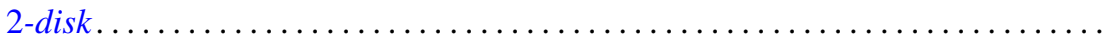

Richard Earl Hodel, Metrizability of topological spaces .................

Wilfried Imrich and Mark E. Watkins, On graphical regular representations of

cyclic extensions of groups .......................... 461

Jozef Krasinkiewicz, Remark on mappings not raising dimension of curves ..... . 479

Melven Robert Krom, Infinite games and special Baire space extensions . . . . . . 483

S. Leela, Stability of measure differential equations . . . . . . . . . . . . . . . . 489

M. H. Lim, Linear transformations on symmetric spaces . . . . . . . . . . . . . . . 499

Teng-Sun Liu, Arnoud C. M. van Rooij and Ju-Kwei Wang, On some group algebra modules related to Wiener's algebra $M_{1} \ldots \ldots \ldots \ldots \ldots \ldots \ldots \ldots \ldots \ldots \ldots$

Dale Wayne Myers, The back-and-forth isomorphism construction ............ 521

Donovan Harold Van Osdol, Extensions of sheaves of commutative algebras by

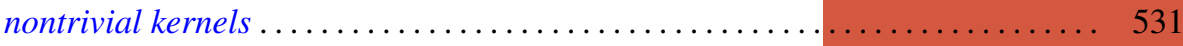

Alan Rahilly, Generalized Hall planes of even order ................... 543

Joylyn Newberry Reed, On completeness and semicompleteness of first countable

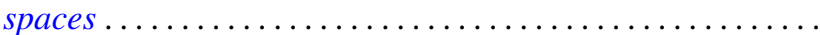

Alan Schwartz, Generalized convolutions and positive definite functions associated

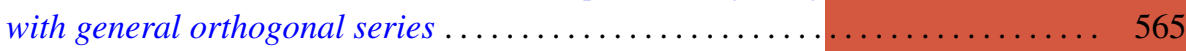

Thomas Jerome Scott, Monotonic permutations of chains . . . . . . . . . . . 583

Eivind Stensholt, An application of Steinberg's construction of twisted groups .... 595

Yasuji Takeuchi, On strongly radicial extensions . . . . ................. 619

William P. Ziemer, Some remarks on harmonic measure in space . . . . . . . . . . 629

John Grant, Corrections to: “Automorphisms definable by formulas” . . . . . . . . 639

Peter Michael Rosenthal, Corrections to: "On an inversion for the general

Mehler-Fock transform pair" ......................... 640

Carl Clifton Faith, Corrections to: "When are proper cyclics injective” . . . . . . 640 